\title{
Prevalence of and risk factors for methicillin-resistant Staphylococcus aureus skin and soft tissue infection in a Canadian emergency department
}

\author{
Robert Stenstrom, MD, PhD; ${ }^{* \ddagger \S}$ Eric Grafstein, MD; ${ }^{* \ddagger \S}$ Marc Romney, MD, MSc; ${ }^{\dagger \S}$ John Fahimi, MD; ${ }^{\S}$ \\ Devin Harris, MD, MSc; ${ }^{* \dagger \S}$ Garth Hunte, MD, MSc; ${ }^{*} \S$ Grant Innes, MD; ${ }^{*}+\S ~ J i m ~ C h r i s t e n s o n, M^{*}+\S$
}

\section{See related articles on pages 417 and 439}

\section{ABSTRACT}

Objective: We sought to estimate the period prevalence of methicillin-resistant Staphylococcus aureus (MRSA) skin and soft tissue infection (SSTI) and evaluate risk factors for MRSA SSTI in an emergency department (ED) population.

Methods: We carried out a cohort study with a nested casecontrol design. Patients presenting to our ED with a wound culture and a discharge diagnosis of SSTI between January 2003 and September 2004 were dichotomized as MRSA positive or negative. Fifty patients with MRSA SSTI matched by calendar time to 100 controls with MRSA-negative SSTI had risk factors assessed using multivariate conditional logistic regression.

Results: Period prevalence of MRSA SSTI was 54.8\% (95\% confidence interval $[\mathrm{Cl}] 50.2 \%-59.4 \%)$. The monthly period prevalence increased from $21 \%$ in January 2003 to $68 \%$ in September 2004 ( $p<0.01$ ). Risk factors for MRSA SSTI were injection drug use (IDU) (odds ratio [OR] 4.6, 95\% Cl 1.4-16.1), previous MRSA infection and colonization (OR 6.4, 95\% Cl 2.1-19.8), antibiotics in 8 weeks preceding index visit (OR 2.6, 95\% Cl 1.2-8.1), diabetes mellitus (OR 4.1, 95\% Cl 1.4-12.1), abscess (OR 5.6, 95\% Cl 1.8-17.1) and admission to hospital in previous 12 months (OR 2.6, 95\% Cl 1.1-11.2).

Conclusion: The period prevalence of MRSA SSTI between January 2003 and September 2004 was 54.8\% at our institution. There was a marked increase in the monthly period prevalence from the beginning to the end of the study. Risk factors are IDU, previous MRSA infection and colonization, prescriptions for antibiotics in previous 8 weeks and admission to hospital in the preceding 12 months. On the basis of local prevalence and risk factor patterns, emergency physicians should consider MRSA as a causative agent for SSTI.
Keywords: methicillin-resistant Staphylococcus aureus (MRSA), skin and soft tissue infection, prevalence, emergency department, risk factors, case-control study

\section{RÉSUMÉ}

Objectif : Nous avons cherché à évaluer la prévalence de période des infections de la peau et des tissus mous (IPTM) à Staphylococcus aureus résistant à la méthicilline (SARM) ainsi que les facteurs de risque associés aux IPTM à SARM auprès d'un groupe de patients d'un service d'urgence.

Méthodes : Nous avons réalisé une étude cas-témoins emboîtée selon un modèle de cohortes. Les patients qui se sont présentés à l'urgence, entre janvier 2003 et septembre 2004, avec une infection ayant fait l'objet d'une culture et qui ont reçu un diagnostic de sortie d'IPTM ont été divisés en cas négatifs ou positifs pour le SRAM. Nous avons évalué, selon une analyse de régression logistique conditionnelle multivariée, les facteurs de risque de 50 patients ayant une IPTM à SARM qui ont été appariés par date à 100 témoins ayant une IPTM non causée par le SARM.

Résultats : La prévalence de période des IPTM à SARM était de $54,8 \%$ (intervalle de confiance [IC] à $95 \%$ de 50,2 à 59,4\%). La prévalence mensuelle est passée de $21 \%$ en janvier 2003 à $68 \%$ en septembre $2004(p<0,01)$. Les facteurs de risque associés aux IPTM à SARM étaient les suivants : utilisation de drogues injectables (UDI) (risque relatif approché [RRA] de 4,6; IC à $95 \%$ de 1,4 à 16,1); infection ou colonisation antérieure à SARM (RRA de 6,4 ; IC à $95 \%$ de 2,1 à 19,8); antibiothérapie dans les 8 semaines précédant la visite à l'urgence (RRA de 2,6; IC à $95 \%$ de 1,2 à 8,1 ), diabète sucré (RRA de 4,1 ; IC à $95 \%$ de 1,4 à 12,1), abcès (RRA de 5,6; IC à $95 \%$ de 1,8 à 17,1) et hospitalisation dans les 12 derniers mois (RRA de 2,6; IC à $95 \%$ de 1,1 à 11,2).

From the *Department of Emergency Medicine, St. Paul's Hospital, Vancouver, BC, the tDepartment of Microbiology, St. Paul's Hospital, Vancouver, BC, $\neq$ The Centre for Health Evaluation and Outcome Sciences, Providence Health Care, Vancouver, BC, and the §Department of Emergency Medicine, Faculty of Medicine, University of British Columbia, Vancouver, BC

Submitted Apr. 29, 2008; Revised Apr. 9, 2009; Accepted Apr. 15, 2009

This article has been peer reviewed. 
Conclusion : La prévalence de période des IPTM à SARM entre janvier 2003 et septembre 2004 était de 54,8 \% à notre établissement. II y a eu une augmentation marquée de la prévalence mensuelle du début à la fin de l'étude. Les facteurs de risque sont I'UDI, une infection ou colonisation antérieure à SARM, une antibiothérapie dans les 8 semaines précédant la visite à l'urgence et I'hospitalisation dans les 12 derniers mois. D'après la prévalence locale et les facteurs de risque, les médecins d'urgence devraient envisager le SARM comme un agent causal (agent étiologique) des IPTM.

\section{INTRODUCTION}

Antibiotic resistance among strains of Staphylococcus aureus (S. aureus) has become an escalating clinical problem in many regions around the world..$^{1-3} S$. aureus was initially sensitive to penicillin, but during the 1960s strains of methicillin-resistant $S$. aureus (MRSA) appeared in nosocomial infections. In the 1990s, reports arose of new strains of community-associated MRSA (CA-MRSA) that were genetically divergent from hospital (or health care)associated MRSA. MRSA causes many infections, such as necrotizing fasciitis, ${ }^{4}$ pneumonia, ${ }^{5}$ meningitis ${ }^{6}$ and endocarditis, ${ }^{7}$ but most frequently it causes skin and soft tissue infection (SSTI), such as cutaneous abscesses, furuncles and cellulitis. ${ }^{8}$ In the last decade, the prevalence of infection caused by MRSA has increased dramatically, ${ }^{1,2}$ as have the associated costs of treatment and infection control. ${ }^{9}$

The increasing frequency of SSTIs caused by MRSA is particularly relevant in the emergency department (ED) both in terms of patient volume and antimicrobial options. More than 2000 patient visits per year are for SSTI at our institution (an inner-city tertiary care ED with about 65000 patient visits per annum). Increasing frequency of MRSA SSTI impacts both antibiotic choices and cost, with increasing use of potent and expensive antimicrobials such as vancomycin.

Identification of risk factors for MRSA SSTI is important for ED patients. Previous studies (mostly in US or European settings) have identified HIV infection and injection drug use (IDU) as risk factors, ${ }^{7}$ as well as incarceration, ${ }^{10}$ and participation in contact sports, such as wrestling. ${ }^{11}$ Clusters of outbreaks have also been reported in isolated Aboriginal communities. ${ }^{12}$

MRSA prevalence and risk factors vary geographically and we were unable to locate previous reports for a Canadian ED population. Increases in the proportion of SSTIs caused by MRSA and risk factors for these infections are important to guide empiric antibiotic therapy and infection site surveillance cultures in the ED. The objectives of this study were to quantify risk factors for MRSA SSTI in an inner-city ED population and to quantify the period prevalence of MRSA SSTI between 2003 and 2005 in a Canadian ED population.
METHODS

\section{Ethics}

This study received approval from the Providence Health Care Ethics Review Board.

\section{Design}

To assess risk factors for MRSA SSTI, we performed a nested case-control study of 50 cases of MRSA SSTI matched on calendar date to 100 controls with SSTI not caused by MRSA. To quantify the period prevalence of MRSA SSTI, we used a cohort study design.

\section{Setting}

The study took place in a tertiary care inner-city ED with approximately 65000 patient visits per year.

\section{Sample size}

The sample size was calculated on the basis of the assessment of risk factors for MRSA SSTI. For an $\alpha$ error rate of 0.05 (2-tailed) to detect an odds ratio (OR) of 2.0 for a given risk factor (assuming a baseline exposure rate of $10 \%$ in controls) and using 2 matched controls for each MRSA SSTI case, 47 case-control sets (141 participants total) were needed for $80 \%$ power based on conditional logistic regression analysis (Power and Precision Statistical Software. V 2.037. Biostat Inc.). ${ }^{13}$ A sample size of 384 participants was required to estimate the $95 \%$ confidence interval (CI) to within 5\% for the period prevalence of MRSA SSTI, assuming a $50 \%$ prevalence. Since we performed 447 wound cultures during this time, we used all of the data for point and interval estimates of period prevalence.

\section{Cohort identification and period prevalence of MRSA SSTI}

Data from the New Emergency Resource Database (NERD) at St. Paul's hospital, Vancouver, BC, revealed that 2234 unique patients were diagnosed with SSTI 
(ICD-9 682.9) between January 2003 and September 2004. These diagnoses included cellulitis, abscess, cellulitis with abscess, furuncle, wound check, impetigo, skin infection not yet diagnosed and wound infection. Infection site cultures from these patients performed in the ED were identified by linking the NERD database to our hospital's microbiology database. Based on infection culture results, all patients were classified as either having an MRSA-positive SSTI or MRSA-negative SSTI. The estimate of period prevalence was based only on those participants who had an infection site culture performed.

We compared period prevalence of discharge diagnoses related to MRSA ("abscess" or "abscess with cellulitis" or "furuncle") versus cellulitis alone between the first 10 months and the last 11 months of the study period for 1931 patients who had any of these diagnoses (the remaining 303 patients had another SSTI diagnosis such as "wound check"). We chose cellulitis as the comparison group, since this diagnosis comprised the majority (more than $90 \%$ ) of the other SSTI diagnoses.

\section{Microbiologic testing and detection of MRSA}

For all infection site cultures following 24-hour incubation, bacterial colonies were picked and further isolated on standard microbiological media for identification and antibiotic susceptibility testing. When necessary, the bacterial gene responsible for methicillin resistance in Stapbylococci $(m e c A)$ was identified by detection of the gene product (PBP2a); alternatively, mecA was detected directly by molecular means using polymerase chain reaction. For molecular typing of MRSA-confirmed isolates, genetic relatedness studies were performed by a reference laboratory using pulsed-field gel electrophoresis.

\section{Case and control selection}

From the 245 patients who had an MRSA-positive SSTI between January 2003 and September 2004, 50 cases were selected using a random number list. One hundred controls ( 2 controls/case) from the 202 patients with MRSA-negative SSTI during the same period were selected. To control for secular trends in risk factor profiles, individual matching of cases and controls was conducted. Controls were individually matched to cases by date of infection site culture $( \pm 2$ mo), and were selected from the 202 patients whose cultures were negative for MRSA. Incident cases were used and controls were able to become cases at a subse- quent point in time if they subsequently developed an MRSA-positive SSTI. Whenever more than 2 possible controls were identified for each case, selection was performed randomly from those eligible. We were able to identify at least 2 time-matched controls for every case.

\section{Risk factor data and definitions}

Data for risk factors for all participants were abstracted by a trained research assistant from 4 sources: the NERD, the electronic order entry database, the printed medical/hospital records and provincial PharmaNet data (for previous antibiotic prescriptions). Based on these sources data were coded and entered into an Excel spreadsheet. Dichotomous variables were coded as positive, negative or not recorded (missing). The research assistant was blinded to case or control status and had 15 randomly selected patient records audited for accuracy. The following definitions for risk factors were used (data source in parentheses).

- Age: age in years dichotomized at 50 years (NERD)

- Sex: male or female (NERD)

- Date of diagnosis: calendar date when infection site culture was obtained (NERD)

- Hospital admission or surgery in previous 12 months: admission to hospital ( $24 \mathrm{~h}$ or longer) and/or surgery requiring a general anasthetic 12 months (or less) preceding date of diagnosis (hospital record)

- Long-term nursing facility: residence in a facility employing 1 or more registered nurse(s) (hospital record)

- Abscess: clinical presence of an abscess as reflected in the discharge diagnosis (i.e., "abscess" or "cellulitis with abscess" or "furuncle") (NERD, hospital record)

- Previous MRSA status: subjects who had a preexisting MRSA alert based on a previous infection (i.e., infection site/blood/CSF/sputum or other fluid culture, or carrier status) (hospital record)

- Injection drug use: patient self-report of IDU on any ED visit, or in past medical records (NERD, hospital record)

- HIV, which was counted as positive if any of the following were positive: hospital virology tests positive for HIV, prescriptions for highly active antiretroviral therapy or self-report (NERD, hospital record).

- Antibiotic prescriptions in previous 8 weeks: based on patient self-report and provincial PharmaNet data, which tracks all prescriptions dispensed for 
residents of British Columbia (NERD, hospital record, PharmaNet)

- Diabetes mellitus: self-report positive and/or patient taking prescription for antihyperglycemic medications, or previously documented diabetes mellitus (NERD, hospital record, PharmaNet)

\section{Statistical methods}

We aimed to determine the period prevalence of SSTIs associated with MRSA. The number of cultures positive for MRSA was divided by the total number of infection site cultures performed between January 2003 and September 2004. A 95\% CI was constructed around the point estimate of period prevalence of MRSA SSTI during the study period. Monthly estimates of period prevalence of MRSA SSTIs were calculated the same way, but on a calendar month-bymonth basis. We used the $\chi^{2}$ test for trend to examine the change in monthly prevalence of MRSA-positive SSTIs during the study period.

Comparison of the period prevalence of SSTI diagnoses related to MRSA (abscess, abscess and cellulitis, or furuncle) for the first 10 months of the study and the last 11 months of the study was conducted using a $\chi^{2}$ test for proportions.
Univariate OR and 95\% CIs for each risk factor were generated using conditional logistic regression. Multivariate analyses were based on conditional logistic regression (matched on calendar date of exposure) to estimate the OR of MRSA SSTI for the risk factors that we measured. We constructed multivariate conditional logistic regression models by including factors that were associated with MRSA in a univariate conditional logistic regression (if $p<0.15$ ). On the basis of biologic plausibility, we initially included age, IDU, admission to hospital in the preceding year and previous MRSA status in the multivariate model along with other risk factors associated with MRSA (univariate $p<0.15$ ). We then removed variables from the model with a backward stepwise procedure $(p$-to-remove $=0.1)$. We assessed two-way interactions between IDU and HIV, and antibiotic prescriptions in the previous 8 weeks and previous MRSA status. Model fit was established using the likelihood ratio test and deviance score. Outlying data points were assessed by plotting Storer-Crowley delta-betas, which estimate how much a regression coefficient changes when an individual case is deleted. Only 5 participants had invasive indwelling devices by our definition, so this variable was not included in the analyses. Egret software (Cytel Inc.) was used for the conditional logistic regression analyses and regression diagnostics.

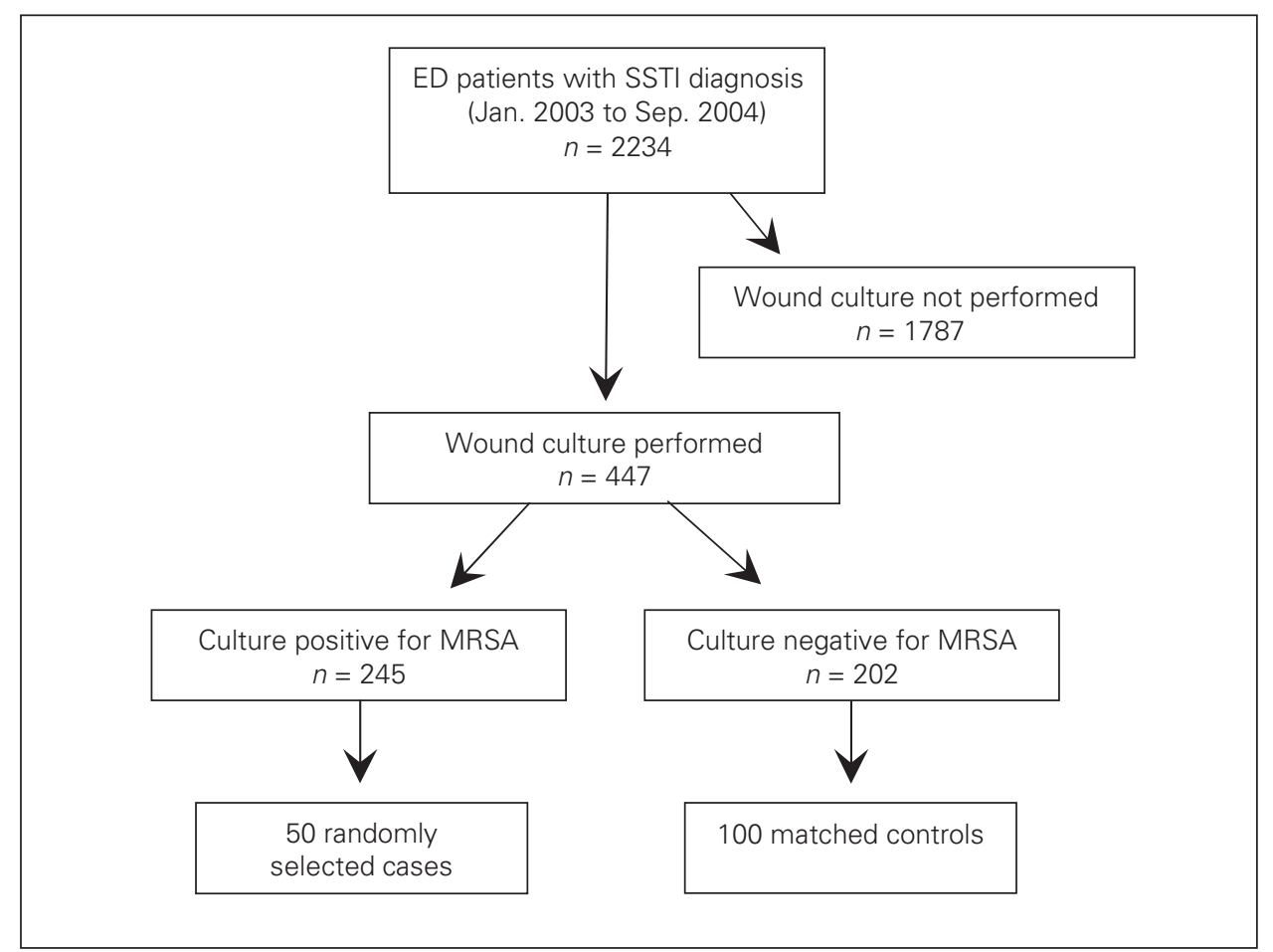

Fig. 1. Derivation of study participants. $E D=$ emergency department; MRSA = methicillinresistant Staphylococcus aureus; SSTI = skin and soft tissue infection. 
Odds ratios were considered statistically significant if the 95\% CI did not include the null value of 1 .

\section{RESULTS}

\section{Participant derivation}

Between January 2003 and September 2004, 2234 patients were diagnosed with SSTI. Of these, 447 (20\%) had a culture performed of their infection site, and these patients formed the basis of the study. We obtained complete data for 150 participants (50 cases each with 2 calendar-time-of-diagnosis-matched controls). Derivation of study participants is shown in Figure 1.

\section{Period prevalence of MRSA SSTI}

Period prevalence of MRSA-positive SSTI during the 21 -month study period was $54.8 \%$ (95\% CI $50.2 \%-$ $59.4 \%)$. The monthly period prevalence of MRSApositive SSTI cultures increased steadily over the 21 -month study period from $21 \%$ of all cultures to $68 \%$ of all cultures at the end of the study period ( $p$ for trend $<0.001)$. Figure 2 arrays these data.
Of the SSTI cultures that were negative for MRSA, 46\% grew methicillin-sensitive S. aureus (MSSA), 25\% grew streptococcal species and $6 \%$ grew various gram negative organisms. Eighteen percent of cultures grew no bacterial organisms. Approximately $5 \%$ of cultures were polymicrobial in controls and $3 \%$ for cases.

\section{Risk factors for MRSA SSTI}

Table 1 summarizes the demographic and risk factor information for cases and controls. Cases and controls were similar in age (mean 48, standard deviation [SD] 18 , yr for cases and 45, SD 16, yr for controls). Intravenous drug use and HIV status were highly correlated. Since IDU had the strongest association with risk of MRSA SSTI, HIV status was removed from the final model.

Statistically significant risk factors for MRSA SSTI were previous MRSA-positive infection or screen (OR 6.4, 95\% CI 2.1-19.8), IDU (OR 4.6, 95\% CI 1.4-16.1), diabetes mellitus (OR 4.1, 95\% CI 1.4-12.1), antibiotic use in the previous 8 weeks (OR 2.6, 95\% CI 1.2-8.1), presence of an abscess (OR 5.6, 95\% CI 1.8-17.1) and admission to hospital in the previous 12 months (OR 2.6, 95\% CI 1.1-11.2). Sex, age,

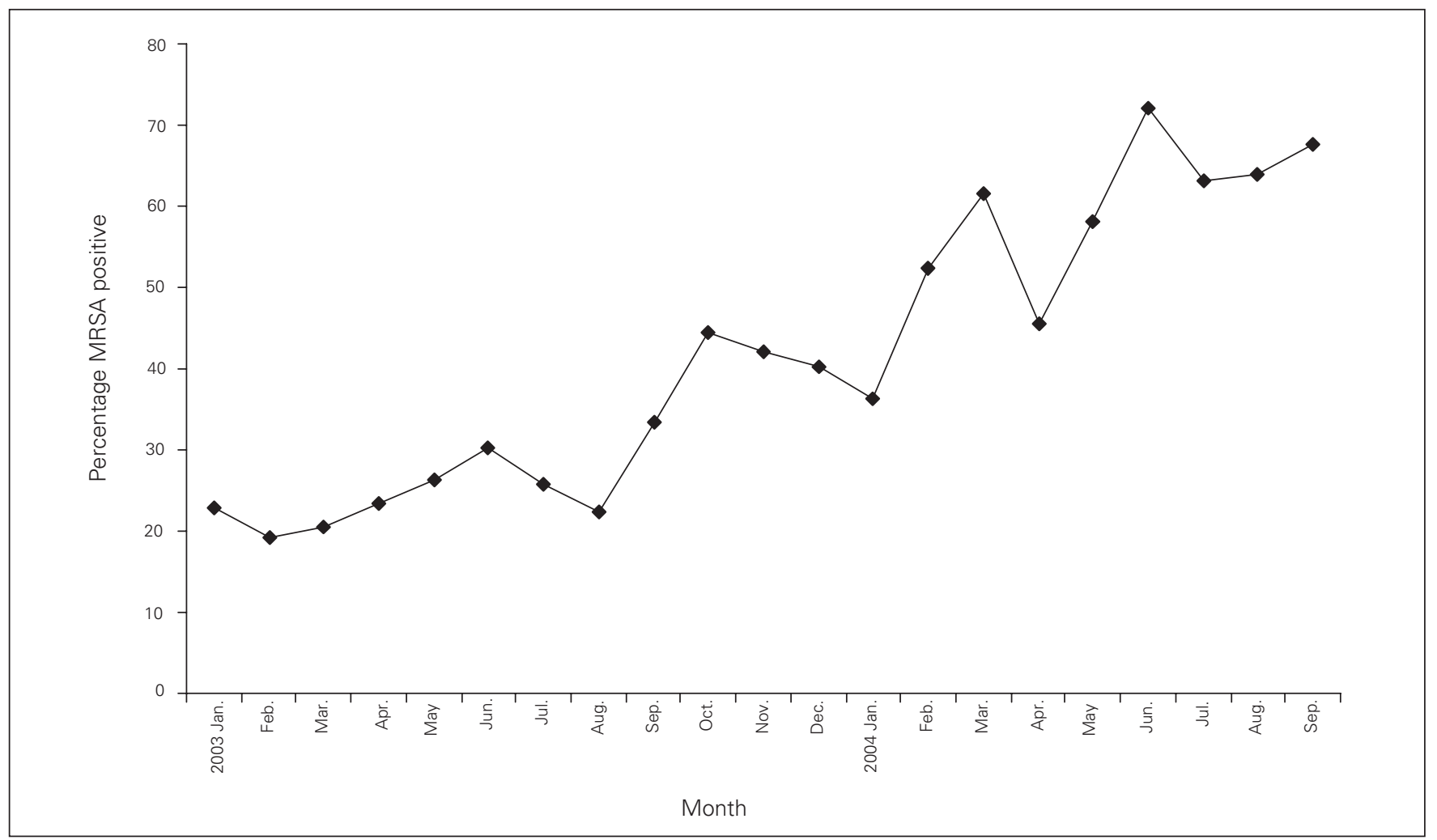

Fig. 2. Percentage of methicillin-resistant Staphylococcus aureus (MRSA)-positive skin and soft tissue infection (SSTI) cultures by month, January 2003-September 2004. 
anatomical area of infection and residence in a longterm care facility were not associated with MRSA SSTI in this data set and were not retained in the final model. Univariate and multivariate ORs and 95\% CIs are shown in Table 2 and Table 3, respectively. The fit of the model with the variables in Table 3 was adequate, based on the likelihood ratio test. Neither of the prespecified 2-way interactions were included in the final model, based on the backward step-wise procedure.

Comparison of the period prevalence of SSTI diagnoses related to MRSA (abscess, abscess and cellulitis, or furuncle) for the first 10 months of the study and the last 11 months of the study revealed a significant increase from $411 / 852(48.2 \%, 95 \%$ CI $44.9 \%-51.5 \%)$ to $691 / 1079$ $(64 \%, 95 \%$ CI $61.1 \%-66.9 \%)\left(\chi^{2}=29.1, p<0.001\right)$.

\section{DISCUSSION}

To our knowledge, this study is the first to report the period prevalence and risk factors for MRSA SSTI in a Canadian ED. Between January 2003 and September 2004 the overall period prevalence of MRSA SSTI was $54.8 \%$, with a pronounced increase in infections linked to MRSA from $22 \%$ to $68 \%$ during the study period.

\begin{tabular}{|c|c|c|c|}
\hline \multirow[b]{2}{*}{ Characteristic } & \multicolumn{3}{|c|}{ No. (\%) of patients } \\
\hline & Cases & $s, n=50$ & Controls, $n=100$ \\
\hline Age $>50 \mathrm{yr}$ & 29 & (58) & 71 (71) \\
\hline Male & 34 & (68) & $52(52)$ \\
\hline Antibiotics in prior 8 weeks & 19 & (38) & $17(17)$ \\
\hline Intravenous drug use & 21 & $(42)$ & $20(20)$ \\
\hline $\begin{array}{l}\text { Hospital admission or } \\
\text { surgery in the previous year }\end{array}$ & 9 & (18) & $8 \quad(8)$ \\
\hline Long-term nursing facility & 2 & (4) & $5 \quad(5)$ \\
\hline HIV positive & 16 & $(32)$ & $14(14)$ \\
\hline Abscess & 36 & $(72)$ & 31 (31) \\
\hline Prior MRSA & 9 & $(18)$ & $5 \quad(5)$ \\
\hline \multicolumn{4}{|l|}{ Anatomical area affected* } \\
\hline Trunk & 7 & (14) & $6 \quad(6)$ \\
\hline Buttocks/perineum & 3 & (6) & $3 \quad(3)$ \\
\hline Upper extremity & 21 & $(42)$ & $46(46)$ \\
\hline Lower extremity & 15 & $(30)$ & 37 (37) \\
\hline Head/neck/face & 3 & (6) & $5 \quad(5)$ \\
\hline Multiple areas & 3 & (6) & $4 \quad(4)$ \\
\hline Diabetes mellitus & 11 & $(22)$ & $6 \quad(6)$ \\
\hline $\begin{array}{l}\text { Permanent indwelling } \\
\text { catheter/medical device }\end{array}$ & 2 & (4) & $3 \quad$ (3) \\
\hline \multicolumn{4}{|c|}{$\begin{array}{l}\text { MRSA = methicillin-resistant Staphylococcus aureus. } \\
\text { *Totals add up to }>10 \% \% \text { because participants were counted more than once if } \\
\text { multiple anatomical areas were affected. }\end{array}$} \\
\hline
\end{tabular}

Although other Canadian data are lacking, our finding is consistent with data from a Los Angeles ED study ${ }^{14}$ that reported that 44/96 (46\%) of infection site cultures from SSTI grew MRSA. A similar study conducted in a San Francisco area $\mathrm{ED}^{8}$ isolated MRSA from $51 \%$ of 137 infection site cultures. A recently published study of 422 patients with SSTI from 11 EDs in the United States reported that the prevalence of MRSA was $59 \% .{ }^{15}$ It is noteworthy that based on data from this study, $71.6 \%$ of $S$. aureus-positive wound infection cultures grew MRSA, and 28.4\% grew MSSA, making MRSA the most common cause of SSTI among cultured patients in our ED. It is striking that the proportion of MRSA-positive infection site cultures from SSTIs increased from $22 \%$ to $68 \%$ during a 21 -month period at our institution. There may be some degree of selection bias reflected in these data since only $20 \%$ of patients with SSTI had infection site cultures performed. The decision to obtain a culture was left up to the discretion of the treating emergency physician, and

Table 2. Univariate odds ratio for risk factors for methicillinresistant Staphylococcus aureus skin and soft tissue infection

\begin{tabular}{lcl} 
Variable & $\begin{array}{c}\text { Crude OR } \\
(95 \% \mathrm{Cl})\end{array}$ \\
\hline Previously MRSA positive & $5.7(2.6-12.8)$ \\
Age $>50 \mathrm{yr}$ & $1.4(0.7-3.7)$ \\
Antibiotics in prior 8 weeks & $2.3(0.8-6.8)$ \\
Male & $1.8(0.9-3.5)$ \\
Intravenous drug use & $2.9(1.2-6.4)$ \\
Hospital admission or surgery in previous year & $5.4(2.3-12.7)$ \\
Abscess & $3.6(1.8-7.3)$ \\
Diabetes mellitus & $6.7(2.4-12.0)$ \\
HIV positive & $3.4(1.3-8.4)$ \\
\hline Cl = confidence interval; MRSA = methicillin-resistant Staphylococcus aureus; &
\end{tabular}

Table 3. Multivariate odds ratios and $95 \%$ confidence intervals for risk markers for methicillin-resistant Staphylococcus aureus skin and soft tissue infection

\begin{tabular}{lc} 
Variable & $\begin{array}{c}\text { Adjusted OR } \\
(95 \% \mathrm{Cl})\end{array}$ \\
\hline Previously MRSA positive & $6.4(2.1-19.8)$ \\
Antibiotic use in prior 8 weeks & $2.6(1.2-8.1)$ \\
Intravenous drug use & $4.6(1.4-16.1)$ \\
Hospital admission or surgery in previous year & $2.6(1.1-11.2)$ \\
Abscess & $5.6(1.8-17.1)$ \\
Diabetes mellitus & $4.1(1.4-12.1)$ \\
\hline $\mathrm{Cl}=$ confidence interval; MRSA = methicillin-resistant Staphylococcus aureus; \\
OR = odds ratio.
\end{tabular}


was likely based on the risk factor profile of the patient and practice pattern of the physician. However, when we compared the breakdown of ED diagnoses during the study period, we observed an increase in the rate of SSTI diagnoses related to MRSA (abscess, abscess and cellulitis, or furuncle), from a period prevalence of $48 \%$ during the first 10 months of the study to $64 \%$ during the last 11 months. Since we examined the diagnoses of all ED patients and not just those with cultures performed, this observation strengthens the likelihood that our observed increase in MRSA-positive cultures was the result of a true increase in prevalence and not because of a change in the rate of cultures.

In the present study, we were unable to differentiate between hospital-associated MRSA and CA-MRSA. After adjusting for other risk factors, admission to hospital or surgery requiring a general anesthetic in the year before the index visit was associated with a 2.6-fold increase in the risk of MRSA SSTI. Recent hospital admission, presence of an indwelling catheter or medical device, and transfer from a skilled nursing facility are accepted risk factors for hospital-associated MRSA. ${ }^{16}$ Thirty-four percent of MRSA cases in this study had 1 or more of these clinical risk factors for hospital-associated MRSA. However, other research has determined that many patients who have risk factors for hospital-associated MRSA, will have a community-associated strain, based on microbiologic testing. ${ }^{8}$

In our study, significant risk factors for MRSA SSTI that could be of clinical utility to the practising emergency physician were as follows: the presence of an abscess (OR 5.6), previous infection or colonization with MRSA (OR 6.4), use of antibiotics in the 8 weeks preceding presentation (OR 2.6), IDU (OR 4.6) and diabetes mellitus (OR 4.1). Other studies have reported a strong association between MRSA and presence of an abscess, or furuncle ${ }^{8}$ or "spider bite." ${ }^{14}$ Communityassociated MRSA frequently carries the gene for Panton-Valentine leukocidin which is directly toxic to neutrophils ${ }^{17}$ and tends to form large and/or numerous cutaneous abscesses. The association between previous MRSA colonization and current MRSA SSTI, as well as recent antibiotic use and MRSA SSTI may reflect a bias of emergency physicians to culture these patients more frequently. However, a recent meta-analysis of over 24000 patients in 76 studies clearly demonstrated an association between exposure to antibiotics and MRSA isolation. ${ }^{18}$ Regardless of etiology, it is entirely appropriate for clinicians to use the presence of these risk factors when prescribing antimicrobial therapy. However, from a therapeutic perspective, it is evident that cutaneous abscesses alone, regardless of cause, do not require antibiotics, but only incision and drainage. ${ }^{19}$ Particularly given the increasing antimicrobial pressure on MRSA, there is growing concern about the possibility of inducible antibiotic resistance in CA-MRSA, particularly for clindamycin, ${ }^{20}$ but this is a laboratory phenomenon and has not been well documented in humans.

Injection drug users were more likely to have MRSA SSTI in this study (OR 4.6). The demographic of our inner-city patient population is reflected by the $20 \%$ of controls and $42 \%$ of cases who reported being injection drug users. In other research, IDU was strongly linked to MRSA SSTI in homeless individuals, ${ }^{7}$ but this association was not found in 2 ED-based studies. ${ }^{8,15}$ There is a possibility that our findings represent a selection bias toward performing infection site cultures more frequently for IDU patients, but it makes biologic sense that frequent intravenous injection under nonsterile conditions increases the risk of infection if the user is already colonized. Similarly, diabetes mellitus was found to be a risk factor for MRSA SSTI (OR 4.1). This association has been reported in another study as well. ${ }^{21}$ It may be that frequent interactions with the health care system lead to increased risk of colonization, where MRSA and diabetic foot ulcers with related breakdown of the skin barrier act as a portal for infection. This study failed to identify HIV status, age, sex or residence in a nursing home as risk factors for MRSA SSTI in this study. HIV status was associated with MRSA SSTI in a univariate analysis, but this relationship was not evident once we adjusted for IDU in the multivariate model.

This study suggests that MRSA SSTI in the ED is increasing in frequency at a rapid rate. Clinicians should consider MRSA as a potential cause of SSTI particularly when the patient has an abscess, uses injection drugs, has had unsuccessful treatment with typical antistreptococcal/ staphylococcal antibiotics, has been MRSA positive previously or has diabetes mellitus. The likelihood of MRSA SSTI increases if the patient has more than 1 risk factor. The issue of which patients with SSTI to culture is controversial; our hospital recently adopted a policy of culturing all SSTIs that have material amenable to culture (i.e., open wounds, abscesses), primarily for reasons of surveillance. A pragmatic approach would be to culture patients who don't respond to the usual treatments. Our practice is to incise and drain all abscesses and to prescribe no antibiotic treatment unless there is a significant cellulitis as well, in which case we also provide the 
patient with a prescription for an oral antibiotic, which covers CA-MRSA (e.g., doxycycline, minocycline, trimethoprim-sulfamethoxazole).

Further research examining prevalence and risk factors for MRSA SSTI in widespread geographic locations is needed. Also, future studies should differentiate between hospital-associated and CA-MRSA and identifiable risk factor profiles for these infections. The need for antibiotic treatment of MRSA abscesses in an ED setting needs to be investigated further since proper incision and drainage alone seems to be a reasonable treatment option.

\section{Limitations}

Our study is limited by the fact that only $20 \%$ of patients with SSTI as a discharge diagnosis had a wound swab performed. The decision to culture a wound was left to the discretion of the emergency physician. Patients with higher risk of MRSA may have had their wounds cultured more frequently, thus overestimating the prevalence of MRSA. However, even if we assume that all of the 1787 patients with SSTI who did not have a wound culture performed were MRSA negative, then the period prevalence of MRSA would still be $11 \%$ (245/2234), making it an important pathogen in SSTI.

The case-control design for the evaluation of risk factors for MRSA SSTI is subject to selection and confounding biases. For example, since many of the IDU population of Vancouver's downtown area use our ED for their primary care, and if they are more prone to SSTI, then the odds ratio for MRSA SSTI associated with IDU will be inflated. By matching MRSA cases with controls by calendar time of wound swab, we were able to control for any secular trends (such as physician practice around obtaining wound swabs) that may have occurred during the study period. Our department's inner-city demographic (homelessness, high proportion of injection drug users, etc.) means that the external validity of our findings may be limited. However, many Canadian EDs are experiencing increases in MRSA SSTI and results from this study regarding independent risk factors other than IDU (e.g., previous MRSA status, diabetes mellitus, recent antibiotic use, hospital admission and presence of an abscess) can be extended to other settings.

We were unable to differentiate community-associated from hospital-associated MRSA. Nevertheless, we suspect that the increase in the prevalence of MRSA SSTI in our ED was largely due to CA-MRSA.

\section{CONCLUSION}

Based on this retrospective study, MRSA is increasing as a cause of SSTI in this Canadian urban ED. The majority of cultures performed in patients with soft tissue infections were MRSA positive at our institution between January 2003 and September 2004, showing a sharp increase during this interval. Risk factors for MRSA SSTI included the presence of an abscess, IDU, previous MRSA status, antibiotic prescriptions within 8 weeks, diabetes mellitus and hospital admission within the preceding year. On the basis of local prevalence and risk factor patterns, emergency physicians should consider MRSA as a causative agent for SSTI.

Competing interests: None declared.

\section{REFERENCES}

1. Elston DM. New and emerging diseases. 7 Am Acad Dermatol 2005;52:1062-8.

2. Eady EA, Cove JH. Staphylococcal resistance revisited: community-acquired methicillin-resistant Staphylococcus aureus - an emerging problem for the management of skin and soft tissue infections. Curr Opin Infect Dis 2003;16:103-24.

3. Nimmo GR, Pearson JC, Collignon PJ, et al. Prevalence of MRSA among Staphylococcus aureus isolated from hospital inpatients, 2005: report from the Australian Group for Antimicrobial Resistance. Commun Dis Intell 2007;31:288-96.

4. Miller LG, Perdreau-Remington GF, Rieg G, et al. Necrotizing fasciitis caused by community-associated methicillinresistant Staphylococcus aureus in Los Angeles. N Engl 7 Med 2005;352:1445-53.

5. Frazee BW, Salz TO, Lambert L, et al. Fatal communityassociated methicillin-resistant Staphylococcus aureus pneumonia in an immunocompetent young adult. Ann Emerg Med 2005;46:401-4.

6. Chang WN, Lu CH, Wu JJ, et al. Staphylococcus aureus meningitis in adults: a clinical comparison of infections caused by methicillin-resistant and methicillin-sensitive strains. Infection 2001;29:245-50.

7. Charlebois ED, Bangsberg DR, Moss NJ, et al. Populationbased community prevalence of methicillin resistant Staphylococcus aureus in the urban poor of San Francisco. Clin Infect Dis 2002;34:425-33.

8. Frazee BW, Lynn J, Charlebois ED, et al. High prevalence of methicillin-resistant Staphylococcus aureus in emergency department skin and soft tissue infections. Ann Emerg Med 2005;45:311-20.

9. Purcell K, Fergie J, Peterson MD. Economic impact of the community-acquired methicillin-resistant Staphylococcus aureus epidemic on the Driscoll Children's Health Plan. Pediatr Infect Dis J 2006;25:178-80.

10. Baillargeon J, Kelley MF, Leach CT, et al. Methicillinresistant Staphylococcus aureus infection in the Texas Prison System. Clin Infect Dis 2004;38:92-5.

11. Lindenmayer JM, Schoenfeld S, O'Grady R, et al. Methicillinresistant Staphylococcus aureus in a high school wrestling 
team and the surrounding community. Arch Intern Med 1998;158:895-9.

12. Groom AV, Wolsey DH, Naimi TS, et al. Communityacquired methicillin-resistant Staphylococcus aureus in a rural American Indian community. JAMA 2001;286:1201-5.

13. Hosmer DW, LeMeshow S. Applied logistic regression. Toronto (ON): John Wiley and Sons; 1989.

14. Moran GJ, Amii RN, Abrahamian FM, et al. Methicillinresistant staphylococcus aureus in community-acquired skin infections. Emerg Infect Dis 2005;11:928-30.

15. Moran GJ, Krishnadasan A, Gorwitz J, et al. Methicillinresistant $S$. aureus infections among patients in the emergency department. N Engl 7 Med 2006;355:666-74.

16. Bartlett M, McIntyre L, Moseley R, et al. Methicillinresistant $\mathrm{S}$. aureus rates increase $260 \%$ in hospitals participating in HIP's international monitoring systems. Centers for Disease Control and National Center for Infectious Diseases Focus 2000;9:4.

17. Genestier AL, Michallet MC, Prevost G, et al. Staphylococcus aureus Panton-Valentine leukocidin directly targets mitochondria and induces Bax-independent apoptosis of human neutrophils. 7 Clin Invest 2005;115:3117-27.

18. Tacconelli E, De Angelis G, Cataldo MA, et al. Does antibi- otic exposure increase the risk of methicillin-resistant Staphylococcus aureus (MRSA) isolation? A systematic review and meta-analysis. J Antimicrob Chemother 2008;61:26-38.

19. Rajendran PM, Young D, Maurer T, et al. Randomized, double-blind, placebo-controlled trial of cephalexin for treatment of uncomplicated skin abscesses in a population at risk for community-acquired methicillin-resistant staphylococcus aureus infection. Antimicrob Agents Chemother 2007;51:4044-8.

20. Chavez-Bueno S, Bozdogan B, Katz K, et al. Inducible clindamycin resistance and molecular epidemiologic trends of pediatric community-acquired methicillin resistant Staphylococcus aureus in Dallas, Texas. Antimicrob Agents Chemother 2005; 49:2283-8.

21. Stanaway S, Johnson D, Moulik P, et al. Methicillin-resistant Staphylococcus aureus (MRSA) isolation from diabetic foot ulcers correlates with nasal MRSA carriage. Diabetes Res Clin Pract 2007;75:47-50.

Correspondence to: Dr. Rob Stenstrom, Emergency Department, St. Paul's Hospital, 1081 Burrard St., Vancouver BC V6Z 1Y6; robstenstrom@shaw.ca

\section{SERVICE INFORMATION}

\section{Subscription and sales}

The Canadian Journal of Emergency Medicine (CJEM) is supplied to paid-up members of the Canadian Association of Emergency Physicians (CAEP) as a perquisite of membership; others may subscribe yearly. Rates for 2009 (6 issues): Canada (personal), \$225; Canadian institutions, \$399; outside Canada (personal), US\$254; US institutions, US\$449. Contact the CAEP office (800 463-1158). Single copies of current year issues \$50; back issues $\$ 50$ (subject to availability). Payment should be made to CAEP in funds specified drawn on a Canadian or US bank. VISA and MasterCard are also accepted.

\section{Change of address}

We require 6-8 weeks' notice to ensure uninterrupted service. Please fax your current mailing label, new address and effective date to 613 523-0190 or email: cjem@caep.ca. Send US and foreign address changes to International Media Services (IMS) of New York, 100 Walnut St., \#3, PO Box 1518, Champlain NY 12919-1518.

\section{Reprints}

Bulk reprints of $C J E M$ articles are available in minimum quantities of 50 . For information or orders, please contact the reprint coordinator, Janis Murrey, $800663-7336$ or 613 731-8610 x2110, fax 613 565-7704, janis.murrey@cma.ca.

\section{Electronic availability}

CJEM is available on the CAEP website (cjem-online.ca).

\section{Indexing}

CJEM is indexed by MEDLINE/PubMed, EMBASE,
CINAHL,International Pharmaceutical Abstracts (IPA), BIOME/OMNI, Scirus, Cochrane Prehospital and Emergency Health Field, and PubsHub.com

\section{Copyright and permissions}

Copyright for all material is held by CJEM or its licensors unless otherwise indicated. You may reproduce or otherwise use material from this Journal only in accordance with Canadian copyright law and provided that credit is given to the original source. In the case of photocopying or other reprographic copying, please contact the Canadian Copyright Licensing Agency (Access Copyright): 800 893-5777; accesscopyright.ca. For any other use, including republishing, redistribution, storage in a retrieval system or transmission, in any form or by any means, please contact the CJEM Editorial Office, c/o Penelope Gray-Allan, Managing Editor, Canadian Journal of Emergency Medicine, Department of Emergency Medicine, University of British Columbia, c/o Department of Emergency Medicine, Vancouver General Hospital, 855 West 12th Ave., Vancouver BC V5Z 1M9; cjem@caep.ca

\section{Instructions for authors}

Published in the January issue of each volume. The most recent version is available online at cjem-online.ca.

\section{Advertising}

Classified ads: Contact Bev Kirkpatrick or Deborah Rodd, CJEM, 1867 Alta Vista Dr., Ottawa ON K1G 5W8; $800663-$ 7336 or 613 731-8610 x2127/2314, fax 613 565-7488, advertising@cma.ca. See Classified Advertising section of the Journal for information on rates. Display ads: Contact Deborah Woodman (see preceding information); x2159. 